\title{
Association of elevated fractional exhaled nitric oxide concentration and blood eosinophil count with severe asthma exacerbations
}

\author{
David B. Price ${ }^{1,2^{*}}$, Sinthia Bosnic-Anticevich ${ }^{3}$, lan D. Pavord ${ }^{4}$, Nicolas Roche ${ }^{5}$, David M. G. Halpin ${ }^{6}$, Leif Bjermer ${ }^{7}$, \\ Omar S. Usmani ${ }^{8}$, Guy Brusselle ${ }^{9}$, Simon Wan Yau Ming ${ }^{1}$ and Sarang Rastogi ${ }^{10}$
}

\begin{abstract}
Background: Blood eosinophil count (BEC) and fractional exhaled nitric oxide (FeNO) concentration are established biomarkers in asthma, associated particularly with the risk of exacerbations. We evaluated the relationship of BEC and FeNO as complementary and independent biomarkers of severe asthma exacerbations.

Methods: This observational study included data from the Optimum Patient Care Research Database. Asthma patients (18-80 years) with valid continuous data for 1 year before FeNO reading, $\geq 1$ inhaled corticosteroid prescription, and $\mathrm{BEC}$ recorded $\leq 5$ years before FeNO reading were separated into cohorts. Categorisation 1 was based on the American Thoracic Society criteria for elevated FeNO concentration (high: $\geq 50 \mathrm{ppb}$; non-high: $<25 \mathrm{ppb}$ ) and BEC (high: $\geq 0.300 \times 10^{9}$ cells/L; non-high: $<0.300 \times 10^{9}$ cells/L). Categorisation 2 (FeNO concentration, high: $\geq 35$ ppb; non-high: $<35 \mathrm{ppb}$ ) was based on prior research. Reference groups included patients with neither biomarker raised.

Results: In categorisation 1, patients with either high FeNO or high BEC $(n=200)$ had a numerically greater exacerbation rate (unadjusted rate ratio, 1.31 [95\% confidence interval: $0.97,1.76]$ ) compared with patients in the reference group. Combination of high FeNO and high BEC $(n=27)$ resulted in a significantly greater exacerbation rate (3.67 $[1.49,9.04])$. Similarly, for categorisation 2 , when both biomarkers were raised $(n=53)$, a significantly greater exacerbation rate was observed $(1.72[1.00,2.93])$.

Conclusion: The combination of high FeNO and high BEC was associated with significantly increased severe exacerbation rates in the year preceding FeNO reading, suggesting that combining FeNO and BEC measurements in primary care may identify asthma patients at risk of exacerbations.
\end{abstract}

Keywords: Asthma, Blood eosinophils, Exhaled airway markers, Nitric oxide

\section{Background}

Asthma, a chronic inflammatory disorder of the airways affecting more than 315 million people worldwide, is associated with considerable morbidity, mortality, and loss of productivity [1-3]. Recognised as a complex, heterogeneous disease, asthma is associated with several phenotypes [4]. Approximately $50 \%$ of all asthma patients demonstrate evidence of eosinophilic airway

\footnotetext{
*Correspondence: dprice@opri.sg

${ }^{2}$ Academic Primary Care, Division of Applied Health Sciences, University of Aberdeen, Polwarth Building, Foresterhill, Aberdeen AB25 2ZD, UK Full list of author information is available at the end of the article
}

inflammation $[5,6]$, which is associated with an increased risk of exacerbations $[7,8]$. Severe asthma exacerbations involve systemic corticosteroid use, emergency room visits, and/or hospitalisations $[9,10]$. Therefore, an important goal in the treatment and management of asthma is preventing exacerbations by identifying patients most at risk.

Blood eosinophil counts and fractional exhaled nitric oxide (FeNO) concentrations are established biomarkers in asthma. A high blood eosinophil count, used as a marker for eosinophilic airway inflammation, correlates well with poor asthma control, an increased risk of severe exacerbations, and re-hospitalisations [11-14]. 
Conversely, a significant reduction in severe exacerbations has been observed for severe asthma patients with elevated blood eosinophils treated with biologics targeting type 2 cytokines involved in eosinophilic inflammation [15-18]. A FeNO concentration greater than 50 parts per billion ( $\mathrm{ppb}$ ) is a marker for eosinophilic airway inflammation and predicts the likelihood of corticosteroid responsiveness $[19,20]$. Moreover, elevated FeNO is considered a risk factor for exacerbations in adult asthma patients prescribed inhaled corticosteroids (ICS) [21, 22]. Therefore, measurement of FeNO may provide additional predictive value to blood eosinophil counts for severe exacerbations in asthma patients.

Although both blood eosinophil count and FeNO concentration are associated with eosinophilic airway inflammation, they demonstrate only a modest correlation, reflecting different parts of the T2-driven inflammation [23-26]. Notably, these biomarkers vary in their responsiveness to and ability to predict response to biologic therapy for asthma $[16,27,28]$.

Anti-interleukin-5 treatment with mepolizumab lowered blood eosinophil counts without affecting FeNO concentrations [28], while blocking interleukin-13 with lebrikizumab reduced FeNO concentrations without affecting blood eosinophil counts [27]. Thus, FeNO may also reflect aspects of T2-driven inflammation not directly related to eosinophils. While strong evidence suggests that ICS treatment has a substantial effect on FeNO readings, sparse evidence supports the doseresponse effect of ICS on blood eosinophil counts [24, 25]. Presence of raised FeNO concentrations and raised blood eosinophil counts, despite adherence to treatment, may identify patients with poor sensitivity to ICS who require a more targeted, personalised approach to therapy. Therefore, identification of a phenotype that demonstrates raised blood eosinophil counts and/or FeNO concentrations, despite ICS therapy, could be valuable. The aim of this study was to determine whether FeNO concentration added value to blood eosinophil counts for identification of patients at risk of asthma exacerbations. We, therefore, retrospectively analysed data from a large validated national database of patients in the United Kingdom (UK) to evaluate whether a high blood eosinophil count combined with high FeNO concentration was associated with an increased risk of severe asthma exacerbations.

\section{Methods}

\section{Data source and study design}

This cross-sectional study was conducted using patient data from the Optimum Patient Care Research Database (OPCRD). The OPCRD is a primary care database containing high-quality anonymised data obtained from longitudinal medical records and patient-completed questionnaires in the UK health care system [29]. Patient data were assessed for 1 year preceding the index date (baseline year). The study was registered under the established study database, namely, the European Network of Centres for Pharmacoepidemiology and Pharmacovigilance (registration number: EUPAS16891). Ethical approvals were obtained from the Anonymised Data Ethics and Protocol Transparency committee [30].

Patients were classified based on their FeNO reading on the index date and the closest blood eosinophil count reading (Fig. 1). Two sets of thresholds were used for FeNO: (1) based on the American Thoracic Society (ATS) [19] criteria defining high FeNO ( $\geq 50 \mathrm{ppb})$, medium FeNO $(25$ to $<50 \mathrm{ppb})$, and low FeNO $(<25 \mathrm{ppb})$ concentrations (categorisation 1); and (2) based on previous research [31, 32] suggesting poor asthma control with FeNO concentrations $\geq 35 \mathrm{ppb}$, high FeNO was defined as $\geq 35 \mathrm{ppb}$ and non-high FeNO, $<35 \mathrm{ppb}$ (categorisation 2 ). In both categorisation schemes, the cutoff to define a high blood eosinophil count was set at $\geq 0.300 \times 10^{9}$ cells/L. Categorisation 1 included three cohorts: high FeNO ( $\geq 50 \mathrm{ppb})$ and high blood eosinophil count $\left(\geq 0.300 \times 10^{9}\right.$ cells $\left./ \mathrm{L}\right)$, high FeNO alone $(\geq 50 \mathrm{ppb})$ or high blood eosinophil count alone $\left(\geq 0.300 \times 10^{9}\right.$ cells/L), and non-high FeNO $(<25 \mathrm{ppb})$ and non-high blood eosinophil count $\left(<0.300 \times 10^{9}\right.$ cells/L) (reference group). Categorisation 2 included four cohorts: high FeNO ( $\geq 35 \mathrm{ppb})$ and high blood eosinophil count $\left(\geq 0.300 \times 10^{9}\right.$ cells/L), high FeNO $(\geq 35 \mathrm{ppb})$ and nonhigh blood eosinophil count $\left(<0.300 \times 10^{9}\right.$ cells/L), nonhigh FeNO $(<35 \mathrm{ppb})$ and high blood eosinophil count $\left(\geq 0.300 \times 10^{9}\right.$ cells $\left./ \mathrm{L}\right)$, and non-high FeNO $(<35 \mathrm{ppb})$ and non-high blood eosinophil count $\left(<0.300 \times 10^{9}\right.$ cells/L) (reference group).

The study period, during which both patient characteristics and outcomes were observed, consisted of the year prior to the latest documented FeNO reading.

\section{Patients}

The study population consisted of patients who met the following criteria: age 18-80 years inclusive; a diagnostic Read code for asthma qualifying for inclusion in the asthma patient registry, which general practices in the UK maintain for the Quality Outcomes Framework [33]; active asthma with $\geq 1$ prescription for asthma medication, including ICS in the year prior to the index date; $\geq 1$ valid blood eosinophil count recorded without a recent exacerbation (within 2 weeks) at most $\leq 5$ years before FeNO reading; and valid continuous data for 1 year prior to the latest FeNO reading.

Patients were excluded from the study if they had a diagnosis Read code for chronic obstructive pulmonary 


\section{Patient subgroups}

\section{Date of} FeNO reading

\section{Categorisation 1}

High FeNO ( $\geq 50 \mathrm{ppb})$ and high blood eosinophil count $\left(\geq 0.300 \times 10^{9} \mathrm{cells} / \mathrm{L}\right)$

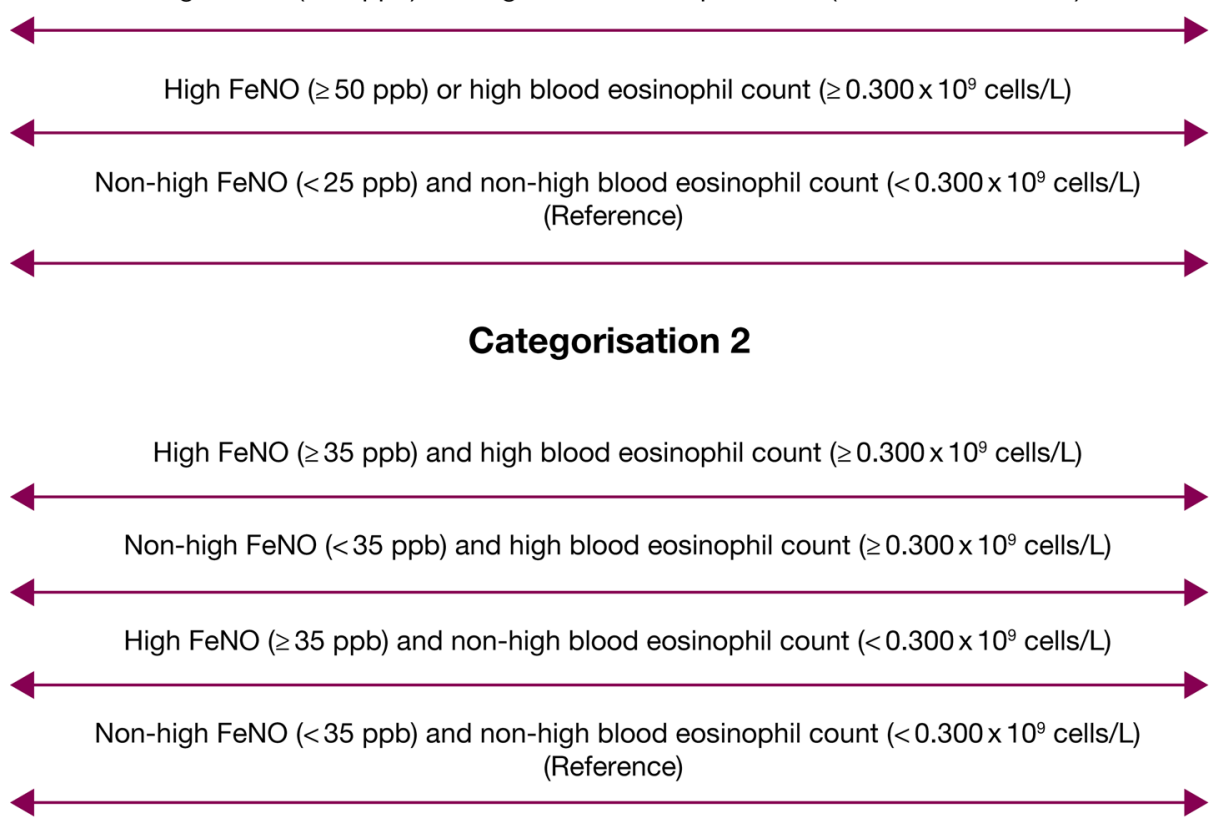

1 year data extraction period

Fig. 1 Study design. The study period consisted of the year prior to the latest documented FeNO reading. FeNO fractional exhaled nitric oxide

disease or any chronic respiratory disease other than asthma; received a long-acting muscarinic antagonist or were prescribed maintenance oral corticosteroids (OCS); and had a forced expiratory volume in $1 \mathrm{~s} /$ forced vital capacity $<0.7$.

\section{Outcomes}

The primary outcome was the annual rate of severe asthma exacerbations, defined as the number of severe exacerbations in the study period per patient. A severe exacerbation was defined in line with the European Respiratory Society/ATS Position Statement [9] as an acute prescription of OCS, or an unplanned lower respiratory tract-related hospitalisation, or an accident and emergency attendance associated with a lower respiratory Read code or primary care respiratory consultation within 14 days.

Secondary outcomes included a description of demographics, lung function, comorbidities, respiratory medication use and ICS adherence for each of the patient groups characterised by biomarker concentrations. ICS adherence was defined using Medication Possession Ratio, calculated by dividing the total of 1 day's supply by the total number of days evaluated (365 days in the study year), and expressed in percentage.

\section{Statistical analyses}

All statistical analyses were conducted using Stata SE version 14.2 and $R$ version 3.0.2.

The sample size was calculated by accounting for multiple testing with a Bonferroni correction. With four comparisons and an alpha significance level of 0.0125, 800 patients were initially deemed necessary to demonstrate at least a $20 \%$ difference between groups, with a $90 \%$ power. However, this was later revised to detect a difference in a single outcome only, namely, a $20 \%$ difference in exacerbation rate between two groups of interest.

Comparisons were initially unmatched for the purpose of exploring the main differences between patient groups and providing the steering committee with data in order to make a decision on which patient groups to compare. In addition, multivariate regression models were fitted to 
account for potential confounding of patient characteristics that may have varied between patient groups. Standardised mean difference was calculated to measure effect size. Characterisation and subsequent matched analyses of study outcomes were performed based on categorisations 1 and 2. Descriptive statistics of all characteristics were computed for each group of patients within the cohorts. Continuous variables were summarised using the number and percentage of non-missing observations, mean and standard deviation (SD) for normally distributed variables, and median and interquartile range (difference between the 25th and 75th percentiles) for non-normally distributed variables. Pearson's Chi square test was used to compare percentages between different groups, with a Fisher's test used in cases of small numbers of observations per group. Student's $t$ test was used to compare a continuous variable between two groups, with a non-parametric Mann-Whitney test used for small numbers of observations per group. Summary statistics were presented as counts and percentages. For missing data, percentages for categorical variables were provided as a percentage of the non-missing observations. A statistically significant result was defined as a $\mathrm{p} \leq 0.05$.

The primary analysis for categorisation 1 compared the number of severe exacerbations for matched patients with a high FeNO and high blood eosinophil count with that of patients with a non-high FeNO and nonhigh blood eosinophil count (reference group). The rate of severe exacerbations was also compared between matched patients with a high FeNO or a high blood eosinophil count vs. the reference group. The analysis for categorisation 2 compared patients with a high FeNO and high blood eosinophil count vs. the reference group, a high blood eosinophil count alone vs. the reference group, and a high FeNO alone vs. the reference group.

Conditional Poisson regression analysis was performed to estimate the rate ratio (RR) between the groups of interest, with a 95\% confidence interval (CI). Unadjusted RRs were calculated based on previous knowledge of multivariable prediction models [34, 35].

\section{Results}

At the time of the study, the OPCRD contained more than 2.4 million available patient records from more than 560 practices across the UK (Fig. 2). According to the records, 1268 patients had a recorded FeNO reading and of these, 610 patients met all other eligibility criteria and were included in the study population. Unmatched comparisons were made to assist with determining the eventual matching criteria. An additional file shows that differences were observed in sex, smoking status, body mass index (BMI), and prescription of OCS in the study year (Additional file 1: Tables S1, S2). Patients were matched 1:1 on age (within 10 years), sex, and smoking status. Further criteria to match were not included to preserve numbers in the cohort of interest.

Patients were subsequently categorised based on FeNO concentration and blood eosinophil count, such that patients from each subgroup with at least one elevated variable of interest (FeNO and/or blood eosinophil count) were matched 1:1 with the reference group (nonhigh FeNO and non-high blood eosinophil count).

\section{Demographics and baseline clinical characteristics Categorisation 1}

A total of 27 patients in the high FeNO and high blood eosinophil cohort matched with the reference group (Table 1). Overall, $63 \%$ of patients were female, with most patients aged 35-65 years. In addition, 51.9\% of patients were non-smokers. In the second cohort, 200 patients with high FeNO or high blood eosinophil count matched with the reference group (Table 2). Overall, $58 \%$ of patients were female, with most patients aged $35-65$ years. A total of $36.5 \%$ of patients were non-smokers.

Demographics and clinical characteristics were generally similar between the matched groups. However, standalone ICS prescriptions were significantly fewer in the high FeNO or high blood eosinophil cohort compared with the reference group (0.6 vs. 1.4 mean standalone ICS prescriptions/patient, $\mathrm{p}=0.0112$ ). Adherence to ICS was comparable between matched groups and was $52.3 \%$ and $63.3 \%$ in the high FeNO and high blood eosinophil cohort and high FeNO or high blood eosinophil cohort, respectively.

\section{Categorisation 2}

Across the biomarker cohorts, more than $50 \%$ of patients were female, with most patients aged 35-65 years. Nonsmokers represented $36-58.5 \%$ of the study sample.

Patients in the non-high FeNO and high blood eosinophil cohort, high FeNO and non-high blood eosinophil cohort, and high FeNO and high blood eosinophil cohort had significantly lower BMI compared with the reference group (29.0 vs. $30.1 \mathrm{~kg} / \mathrm{m}^{2}, \mathrm{p}=0.0492 ; 26.9$ vs. $29.3 \mathrm{~kg} /$ $\mathrm{m}^{2}, \mathrm{p}=0.0063$; and 26.8 vs. $29.0 \mathrm{~kg} / \mathrm{m}^{2}, \mathrm{p}=0.0386$, respectively). All other baseline demographics were well-balanced between the matched groups (Tables 3, 4, 5). For comorbidities, a greater number of patients had a diagnosis of rhinitis in the non-high FeNO and high blood eosinophil cohort compared with the reference group ( 88 vs. 67 patients, $p=0.0272$ ). In addition, differences were observed in the number of ICS prescriptions per patient. Patients in the non-high FeNO and high blood eosinophil cohort, as well as the high FeNO and 


\begin{tabular}{|c|c|}
\hline $\begin{array}{l}\text { OPCRD patients at time of data extraction } \\
\qquad \mathrm{n}=2,423,445\end{array}$ & Exclusion criteria \\
\hline Patients with FeNO reading recorded & $\begin{array}{l}\text { No FeNO readings } \\
n=2,422,177\end{array}$ \\
\hline $\begin{array}{l}\text { Asthma diagnosis or evidence } \\
\text { of treatment with no COPD } \\
\qquad n=1120\end{array}$ & $\begin{array}{l}\text { No asthma diagnosis or } \\
\text { evidence of treatment } \\
n=148\end{array}$ \\
\hline $\begin{array}{l}\geq 1 \text { ICS prescription } \\
\qquad n=1015\end{array}$ & $\begin{array}{l}\text { No ICS prescription in } 1 \text { year } \\
\text { before FeNO reading } \\
n=105\end{array}$ \\
\hline $\begin{array}{l}\text { Age between } 18 \text { and } 80 \text { years (inclusive) } \\
\qquad n=822\end{array}$ & $\begin{array}{c}\text { Aged }<18 \text { or }>80 \text { years } \\
\text { at time of FeNO reading } \\
n=193\end{array}$ \\
\hline $\begin{array}{l}\text { Blood eosinophil reading within } \\
5 \text { years before index date } \\
n=656\end{array}$ & $\begin{array}{l}\text { No blood eosinophil reading } \\
5 \text { years before FeNO reading } \\
\qquad n=166\end{array}$ \\
\hline $\begin{array}{l}\geq 1 \text { year of data before FeNO reading } \\
\qquad n=637\end{array}$ & $\begin{array}{l}<1 \text { year of data before FeNO reading } \\
\qquad n=19\end{array}$ \\
\hline $\begin{array}{l}\text { No other chronic respiratory condition } \\
\qquad n=621\end{array}$ & $\begin{array}{l}\text { Other chronic respiratory conditions } \\
\qquad n=16\end{array}$ \\
\hline $\begin{array}{l}\text { No maintenance OCS } \\
\qquad n=612\end{array}$ & $\begin{array}{l}\text { Maintenance OCS use } \\
n=9\end{array}$ \\
\hline $\begin{array}{l}\text { FeNO reading within expected range } \\
\qquad n=610\end{array}$ & $\begin{array}{l}\text { FeNO reading outside } \\
\text { of expected range } \\
n=2\end{array}$ \\
\hline
\end{tabular}

Fig. 2 Patient selection from OPCRD. COPD chronic obstructive pulmonary disease, FeNO fractional exhaled nitric oxide, ICS inhaled corticosteroid, OCS oral corticosteroid, OPCRD Optimum Patient Care Research Database

non-high blood eosinophil cohort, had fewer standalone ICS prescriptions per patient relative to the reference group (0.7 vs. 1.3 mean standalone ICS prescriptions/ patient, $\mathrm{p}=0.0362$ and 0.9 vs. $1.6, \mathrm{p}=0.0295$, respectively). Adherence to ICS was not significantly different between matched groups and was $66.2 \%, 65.7 \%$, and $68.6 \%$ in the non-high FeNO and high blood eosinophil, high FeNO and non-high blood eosinophil, and high FeNO and high blood eosinophil cohorts, respectively.

\section{Asthma Exacerbations Categorisation 1}

In the high FeNO and high blood eosinophil count cohort, a significantly greater percentage of patients were in the greater exacerbation categories compared with patients in the reference group $(\mathrm{p}=0.0427)$ (Additional file 1: Table S3). The mean (SD) number of exacerbations was also significantly greater relative to the reference group (0.8 [1.0] vs. 0.2 [0.4]; $\mathrm{p}=0.0109)$. Overall, the estimated rate of exacerbations in the high FeNO and high blood eosinophil cohort was statistically significantly greater (unadjusted RR: 3.67 [95\% CI: 1.49, 9.04], $\mathrm{p}=0.005)$ compared with matched patients in the reference group (Fig. 3). Likewise, significantly more patients were in the greater exacerbation categories in the high FeNO or high blood eosinophil cohort compared with patients in the reference group $(\mathrm{p}=0.0481)$; however, the mean (SD) number of exacerbations was not significantly different from that in the reference group $(0.5[0.8]$ vs. 0.4 [0.6]; $\mathrm{p}=0.3423$ ) (Additional file 1: Table S3). Overall, the exacerbation rate was numerically greater but did not reach statistical significance when compared with matched patients in the reference group (1.31 [95\% CI: $0.97,1.76], \mathrm{p}=0.081$ ).

\section{Categorisation 2}

In both the non-high FeNO and high blood eosinophil cohort and the high FeNO and non-high blood 
Table 1 Categorisation 1: non-high FeNO and non-high blood eosinophils vs. high FeNO and high blood eosinophils

\begin{tabular}{|c|c|c|c|}
\hline Characteristics & $\begin{array}{l}\text { Non-high FeNO and non-high blood } \\
\text { eosinophils }(n=27)\end{array}$ & $\begin{array}{l}\text { High FeNO and high blood } \\
\text { eosinophils }(n=27)\end{array}$ & $\mathrm{p}$-value \\
\hline \multicolumn{4}{|l|}{ Sex } \\
\hline n (\% non-missing) & $27(100.0)$ & $27(100.0)$ & 1.0000 \\
\hline Male & $10(37.0)$ & $10(37.0)$ & \\
\hline \multicolumn{4}{|l|}{ Age } \\
\hline n (\% non-missing) & $27(100.0)$ & $27(100.0)$ & 0.9379 \\
\hline Mean (SD) & $43.5(18.8)$ & $43.3(19.0)$ & \\
\hline Median (IQR) & $41.0(37.0)$ & $41.0(37.0)$ & \\
\hline \multicolumn{4}{|l|}{ Age group } \\
\hline n (\% non-missing) & $27(100.0)$ & $27(100.0)$ & 1.0000 \\
\hline Under 35 & $12(44.4)$ & $12(44.4)$ & \\
\hline $35-65$ & $9(33.3)$ & $9(33.3)$ & \\
\hline $66-80$ & $6(22.2)$ & $6(22.2)$ & \\
\hline \multicolumn{4}{|l|}{ Smoking status } \\
\hline n (\% non-missing) & $27(100.0)$ & $27(100.0)$ & 0.7645 \\
\hline Non-smoker & $13(48.1)$ & $14(51.9)$ & \\
\hline Ex-smoker & $1(3.7)$ & $2(7.4)$ & \\
\hline Current smoker & $13(48.1)$ & $11(40.7)$ & \\
\hline \multicolumn{4}{|l|}{ BMI } \\
\hline n (\% non-missing) & $23(85.2)$ & $26(96.3)$ & 0.4346 \\
\hline Mean (SD) & $27.7(7.2)$ & $26.9(6.4)$ & \\
\hline Median (IQR) & $26.8(5.5)$ & $25.5(8.4)$ & \\
\hline \multicolumn{4}{|c|}{ Active eczema diagnosis $^{a}$} \\
\hline n (\% non-missing) & $27(100.0)$ & $27(100.0)$ & 0.6387 \\
\hline Yes & $3(11.1)$ & $2(7.4)$ & \\
\hline \multicolumn{4}{|c|}{ Active rhinitis diagnosis $^{\mathrm{a}}$} \\
\hline n (\% non-missing) & $27(100.0)$ & $27(100.0)$ & 0.2482 \\
\hline Yes & $7(25.9)$ & $11(40.7)$ & \\
\hline \multicolumn{4}{|l|}{ Eczema diagnosis } \\
\hline n (\% non-missing) & $27(100.0)$ & $27(100.0)$ & 0.7801 \\
\hline Yes & $10(37.0)$ & $11(40.7)$ & \\
\hline \multicolumn{4}{|l|}{ Rhinitis diagnosis } \\
\hline n (\% non-missing) & $27(100.0)$ & $27(100.0)$ & 0.1628 \\
\hline Yes & $8(29.6)$ & $13(48.1)$ & \\
\hline \multicolumn{4}{|l|}{ IHD diagnosis } \\
\hline n (\% non-missing) & $27(100.0)$ & $27(100.0)$ & 0.5525 \\
\hline Yes & $2(7.4)$ & $1(3.7)$ & \\
\hline \multicolumn{4}{|l|}{ Heart failure diagnosis } \\
\hline n (\% non-missing) & $27(100.0)$ & $27(100.0)$ & \\
\hline Yes & $0(0.0)$ & $0(0.0)$ & \\
\hline \multicolumn{4}{|c|}{ Hypertension diagnosis } \\
\hline n (\% non-missing) & $27(100.0)$ & $27(100.0)$ & 0.4436 \\
\hline Yes & $5(18.5)$ & $3(11.1)$ & \\
\hline \multicolumn{4}{|l|}{ Diabetes diagnosis } \\
\hline n (\% non-missing) & $27(100.0)$ & $27(100.0)$ & \\
\hline Yes & $0(0.0)$ & $0(0.0)$ & \\
\hline \multicolumn{4}{|l|}{ GERD active diagnosis } \\
\hline n (\% non-missing) & $27(100.0)$ & $27(100.0)$ & 0.3128 \\
\hline Yes & $1(3.7)$ & $0(0.0)$ & \\
\hline
\end{tabular}


Table 1 (continued)

\begin{tabular}{|c|c|c|c|}
\hline Characteristics & $\begin{array}{l}\text { Non-high FeNO and non-high blood } \\
\text { eosinophils }(n=27)\end{array}$ & $\begin{array}{l}\text { High FeNO and high blood } \\
\text { eosinophils }(n=27)\end{array}$ & p-value \\
\hline \multicolumn{4}{|l|}{ Predicted peak flow } \\
\hline n (\% non-missing) & $14(51.9)$ & $20(74.1)$ & \multirow[t]{3}{*}{0.9721} \\
\hline Mean (SD) & $488.9(53.4)$ & $505.8(74.9)$ & \\
\hline Median (IQR) & $482.7(47.2)$ & $478.3(127.7)$ & \\
\hline \multicolumn{4}{|c|}{ ICS/LABA prescriptions per patient } \\
\hline n (\% non-missing) & $27(100.0)$ & $27(100.0)$ & \multirow[t]{3}{*}{0.6546} \\
\hline Mean (SD) & $3.6(4.3)$ & $3.2(2.5)$ & \\
\hline Median (IQR) & $1.0(7.0)$ & $3.0(5.0)$ & \\
\hline \multicolumn{4}{|c|}{ Mono ICS prescriptions per patient } \\
\hline n (\% non-missing) & $27(100.0)$ & $27(100.0)$ & \multirow[t]{3}{*}{0.4898} \\
\hline Mean (SD) & $1.2(1.9)$ & $1.0(2.2)$ & \\
\hline Median (IQR) & $0.0(2.0)$ & $0.0(1.0)$ & \\
\hline \multicolumn{4}{|c|}{ Mean daily SABA dosage $(\mu \mathrm{g})$} \\
\hline n (\% non-missing) & $27(100.0)$ & $27(100.0)$ & \multirow[t]{5}{*}{0.5066} \\
\hline$<100$ & $8(29.6)$ & $6(22.2)$ & \\
\hline $100-200$ & $11(40.7)$ & $8(29.6)$ & \\
\hline $201-400$ & $6(22.2)$ & $8(29.6)$ & \\
\hline$>400$ & $2(7.4)$ & $5(18.5)$ & \\
\hline \multicolumn{4}{|l|}{ ICS adherence ${ }^{b}$} \\
\hline n (\% non-missing) & $27(100.0)$ & $27(100.0)$ & \multirow[t]{3}{*}{0.7158} \\
\hline Mean (SD) & $57.1(39.3)$ & $52.3(32.9)$ & \\
\hline Median (IQR) & $54.8(54.8)$ & $49.3(35.7)$ & \\
\hline
\end{tabular}

All values in the table are $\mathrm{n}(\%)$ unless otherwise specified. High FeNO defined as $\geq 50 \mathrm{ppb}$; non-high FeNO $<25$ ppb; high blood eosinophil count defined as $\geq 0.300 \times 10^{9}$ cells $/ \mathrm{L}$; non-high blood eosinophil count $<0.300 \times 10^{9}$ cells $/ \mathrm{L}$

ATS American Thoracic Society, BMI body mass index, FeNO fractional exhaled nitric oxide, GERD gastroesophageal reflux disease, ICS inhaled corticosteroid, IHD ischaemic heart disease, IQR interquartile range, $L A B A$ long-acting $\beta_{2}$-agonist, $p p b$ parts per billion, SABA short-acting $\beta_{2}$-agonist, $S D$ standard deviation

a Active denotes diagnosed in the year before FeNO reading or treated in the year before FeNO reading

b Medication Possession Ratio was calculated by dividing the total of 1 day's supply by the total number of days evaluated, multiplied by $100 \%$. The evaluation period for all patients was 365 days in the study year

eosinophil cohort, the mean number of exacerbations was not significantly different from those for the reference groups $(0.5[0.9]$ vs. $0.4[0.7], \mathrm{p}=0.3134$ and 0.5 [0.7] vs. $0.3[0.6], \mathrm{p}=0.1332$, respectively) (Additional file 1: Table S3). While both groups demonstrated a clear trend towards greater exacerbation rates (1.41 [95\% CI: 0.91, 2.19], $\mathrm{p}=0.124$ and 1.35 [95\% CI: $0.99,1.84], \mathrm{p}=0.054$, respectively) in comparison with the reference group, this did not reach statistical significance (Fig. 3). For the high FeNO and high blood eosinophil cohort (both biomarkers elevated), although the mean number of exacerbations was not significantly different from that in the reference group (0.7 [0.9] vs. 0.4 [0.7], $\mathrm{p}=0.116$ ) (Additional file 1: Table S3), a significantly greater exacerbation rate was observed (1.72 [95\% CI: 1.00, 2.93], $\mathrm{p}=0.050)$ compared with the reference group (Fig. 3).

\section{Discussion}

With the development of new biologics that target eosinophilic airway inflammation, accurate and easy-to-use biomarkers to predict asthma exacerbations and likely patient responses to treatment are needed. We conducted a real-world matched cohort study to investigate the relationship between blood eosinophil count, FeNO readings, and the severe exacerbation rate observed in asthma patients prescribed ICS.

We observed that for categorisation 1, based on ATS criteria for FeNO cutoffs, patients with a high FeNO $(\geq 50 \mathrm{ppb})$ and high blood eosinophil count $\left(\geq 0.300 \times 10^{9}\right.$ cells/L) were almost four-times as likely to have had a severe exacerbation compared with patients with non-high FeNO $(<25 \mathrm{ppb})$ and non-high blood eosinophil count $\left(<0.300 \times 10^{9}\right.$ cells/L) in the year preceding the FeNO reading. In patients with either a high 
Table 2 Categorisation 1: non-high FeNO and non-high blood eosinophils vs. high FeNO or high blood eosinophils

\begin{tabular}{|c|c|c|c|}
\hline Characteristics & $\begin{array}{l}\text { Non-high FeNO and non-high blood } \\
\text { eosinophils }(n=200)\end{array}$ & $\begin{array}{l}\text { High FeNO or high blood eosinophils } \\
(n=200)\end{array}$ & p-value \\
\hline \multicolumn{4}{|l|}{ Sex } \\
\hline n (\% non-missing) & $200(100.0)$ & $200(100.0)$ & \multirow[t]{2}{*}{1.0000} \\
\hline Male & $84(42.0)$ & $84(42.0)$ & \\
\hline \multicolumn{4}{|l|}{ Age } \\
\hline n (\% non-missing) & $200(100.0)$ & $200(100.0)$ & \multirow[t]{3}{*}{0.9223} \\
\hline Mean (SD) & $51.7(13.1)$ & $51.6(13.2)$ & \\
\hline Median (IQR) & $54.0(18.5)$ & $53.0(19.5)$ & \\
\hline \multicolumn{4}{|l|}{ Age group } \\
\hline n (\% non-missing) & $200(100.0)$ & $200(100.0)$ & \multirow[t]{4}{*}{0.4289} \\
\hline Under 35 & $24(12.0)$ & $25(12.5)$ & \\
\hline $35-65$ & $150(75.0)$ & $140(70.0)$ & \\
\hline $66-80$ & $26(13.0)$ & $35(17.5)$ & \\
\hline \multicolumn{4}{|l|}{ Smoking status } \\
\hline n (\% non-missing) & $200(100.0)$ & $200(100.0)$ & \multirow[t]{4}{*}{1.0000} \\
\hline Non-smoker & 73 (36.5) & $73(36.5)$ & \\
\hline Ex-smoker & $23(11.5)$ & $23(11.5)$ & \\
\hline Current smoker & $71(35.5)$ & $71(35.5)$ & \\
\hline \multicolumn{4}{|l|}{ BMl } \\
\hline n (\% non-missing) & $189(94.5)$ & $191(95.5)$ & \multirow[t]{3}{*}{0.1025} \\
\hline Mean (SD) & $30.0(6.9)$ & $29.1(7.0)$ & \\
\hline Median (IQR) & $28.7(8.1)$ & $27.8(7.9)$ & \\
\hline \multicolumn{4}{|c|}{ Active eczema diagnosis $^{a}$} \\
\hline n (\% non-missing) & $200(100.0)$ & $200(100.0)$ & \multirow[t]{2}{*}{0.3347} \\
\hline Yes & $7(3.5)$ & $11(5.5)$ & \\
\hline \multicolumn{4}{|c|}{ Active rhinitis diagnosis $^{a}$} \\
\hline n (\% non-missing) & $200(100.0)$ & $200(100.0)$ & \multirow[t]{2}{*}{0.1056} \\
\hline Yes & $55(27.5)$ & $70(35.0)$ & \\
\hline \multicolumn{4}{|l|}{ Eczema diagnosis } \\
\hline n (\% non-missing) & $200(100.0)$ & $200(100.0)$ & \multirow[t]{2}{*}{0.1284} \\
\hline Yes & $54(27.0)$ & $68(34.0)$ & \\
\hline \multicolumn{4}{|l|}{ Rhinitis diagnosis } \\
\hline n (\% non-missing) & $200(100.0)$ & $200(100.0)$ & \multirow[t]{2}{*}{0.0858} \\
\hline Yes & 77 (38.5) & $94(47.0)$ & \\
\hline \multicolumn{4}{|l|}{ IHD diagnosis } \\
\hline n (\% non-missing) & $200(100.0)$ & $200(100.0)$ & \multirow[t]{2}{*}{1.0000} \\
\hline Yes & $9(4.5)$ & $9(4.5)$ & \\
\hline \multicolumn{4}{|l|}{ Heart failure diagnosis } \\
\hline n (\% non-missing) & $200(100.0)$ & $200(100.0)$ & \multirow[t]{2}{*}{0.3167} \\
\hline Yes & $0(0.0)$ & $1(0.5)$ & \\
\hline \multicolumn{4}{|c|}{ Hypertension diagnosis } \\
\hline n (\% non-missing) & $200(100.0)$ & $200(100.0)$ & 0.0592 \\
\hline Yes & $55(27.5)$ & $39(19.5)$ & \\
\hline Diabetes diagnosis & & & \\
\hline n (\% non-missing) & $200(100.0)$ & $200(100.0)$ & 0.8630 \\
\hline Yes & $19(9.5)$ & $18(9.0)$ & \\
\hline GERD active diagnosi & & & \\
\hline n (\% non-missing) & $200(100.0)$ & $200(100.0)$ & 0.0630 \\
\hline Yes & $35(17.5)$ & $22(11.0)$ & \\
\hline
\end{tabular}


Table 2 (continued)

\begin{tabular}{|c|c|c|c|}
\hline Characteristics & $\begin{array}{l}\text { Non-high FeNO and non-high blood } \\
\text { eosinophils }(n=200)\end{array}$ & $\begin{array}{l}\text { High FeNO or high blood eosinophils } \\
(n=200)\end{array}$ & p-value \\
\hline \multicolumn{4}{|l|}{ Predicted peak flow } \\
\hline n (\% non-missing) & $105(52.5)$ & $110(55.0)$ & \multirow[t]{3}{*}{0.7422} \\
\hline Mean (SD) & $516.0(73.2)$ & $519.4(75.8)$ & \\
\hline Median (IQR) & $485.8(134.7)$ & $487.6(137.8)$ & \\
\hline \multicolumn{4}{|c|}{ ICS/LABA prescriptions per patient } \\
\hline n (\% non-missing) & $200(100.0)$ & $200(100.0)$ & \multirow[t]{3}{*}{0.4736} \\
\hline Mean (SD) & $4.1(4.0)$ & $4.1(3.7)$ & \\
\hline Median (IQR) & $3.0(5.0)$ & $3.5(5.0)$ & \\
\hline \multicolumn{4}{|c|}{ Mono ICS prescriptions per patient } \\
\hline n (\% non-missing) & $200(100.0)$ & $200(100.0)$ & \multirow[t]{3}{*}{0.0112} \\
\hline Mean (SD) & $1.4(2.7)$ & $0.6(1.6)$ & \\
\hline Median (IQR) & $0.0(1.0)$ & $0.0(0.0)$ & \\
\hline \multicolumn{4}{|c|}{ Mean daily SABA dosage $(\mu \mathrm{g})$} \\
\hline n (\% non-missing) & $200(100.0)$ & $200(100.0)$ & \multirow[t]{5}{*}{0.2808} \\
\hline$<100$ & $67(33.5)$ & $83(41.5)$ & \\
\hline $100-200$ & $58(29.0)$ & $47(23.5)$ & \\
\hline $201-400$ & $45(22.5)$ & $47(23.5)$ & \\
\hline$>400$ & $30(15.0)$ & $23(11.5)$ & \\
\hline \multicolumn{4}{|l|}{ ICS adherence ${ }^{b}$} \\
\hline n (\% non-missing) & $200(100.0)$ & $200(100.0)$ & \multirow[t]{3}{*}{0.1931} \\
\hline Mean (SD) & $72.2(72.7)$ & $63.3(53.3)$ & \\
\hline Median (IQR) & $61.7(64.4)$ & $52.0(61.7)$ & \\
\hline
\end{tabular}

All values in table are $\mathrm{n}(\%)$ unless otherwise specified. High FeNO defined as $\geq 50 \mathrm{ppb}$; non-high FeNO $<25$ ppb; high blood eosinophil count defined as $\geq 0.300 \times 10^{9}$ cells/L; non-high blood eosinophil count $<0.300 \times 10^{9}$ cells $/ \mathrm{L}$

ATS American Thoracic Society, BMI body mass index, FeNO fractional exhaled nitric oxide, GERD gastroesophageal reflux disease, ICS inhaled corticosteroid, IHD ischaemic heart disease, IQR interquartile range, $L A B A$ long-acting $\beta_{2}$-agonist, $p p b$ parts per billion, $S A B A$ short-acting $\beta_{2}$-agonist, $S D$ standard deviation

a Active denotes diagnosed in the year before FeNO reading or treated in the year before FeNO reading

b Medication Possession Ratio was calculated by dividing the total of 1 day's supply by the total number of days evaluated, multiplied by $100 \%$. The evaluation period for all patients was 365 days in the study year

FeNO reading or a high blood eosinophil count, the exacerbation RR was less pronounced and non-significant compared with the reference group. In categorisation 2, patients in the high FeNO (>35 ppb) and high eosinophil count $\left(\geq 0.300 \times 10^{9}\right.$ cells/L) cohort were almost twice as likely to have severe exacerbations in the year prior to the FeNO reading compared with the reference group, whereas the high FeNO and non-high blood eosinophil count cohort and non-high FeNO and high blood eosinophil count cohort displayed a trend towards increased exacerbations relative to the reference group, that did not reach statistical significance. Therefore, the combination of blood eosinophil count and FeNO may be an even stronger marker of exacerbation risk compared with the individual biomarkers. Moreover, the use of the ATS criteria for high FeNO ( $\geq 50 \mathrm{ppb})$ resulted in a greater estimated exacerbation rate, indicating that a greater FeNO reading ( $\geq 50 \mathrm{ppb}$ vs. $\geq 35 \mathrm{ppb}$ ) in the presence of a raised blood eosinophil count was associated with an even greater exacerbation rate. Notably, the exacerbation risk seemed to be independent of traditionally used prognostic variables such as predicted peak flow and short-acting $\beta_{2}$-agonist use, which were not significantly different between cohorts.

The cutoffs used in the study to define high FeNO concentration and high blood eosinophil count warrant further consideration. The cutoff chosen for high blood eosinophil count $\left(\geq 0.300 \times 10^{9}\right.$ cells/L) was well within the range of peripheral blood eosinophils (usually ranging between $0.200 \times 10^{9}$ cells $/ \mathrm{L}$ and $0.300 \times 10^{9}$ cells $/ \mathrm{L}$ ) that most accurately predicts sputum eosinophil count in patients with severe asthma [36]. For FeNO classification, the ATS criteria for adults is commonly used, wherein the high FeNO cutoff has been set at $>50 \mathrm{ppb}$ and low FeNO at $<25 \mathrm{ppb}[19,23,37]$. As cutoff concentrations for high, medium, and low FeNO may be confusing for clinicians with relatively little experience of FeNO as a biomarker, we tested a simplified FeNO cutoff criteria (high FeNO, $\geq 35 \mathrm{ppb}$; non-high $\mathrm{FeNO},<35 \mathrm{ppb}$ ) for ease of use in primary care settings. 
Table 3 Categorisation 2: non-high FeNO and non-high blood eosinophils vs. non-high FeNO and high blood eosinophils

\begin{tabular}{|c|c|c|c|}
\hline Characteristics & $\begin{array}{l}\text { Non-high FeNO and non-high blood } \\
\text { eosinophils }(n=186)\end{array}$ & $\begin{array}{l}\text { Non-high FeNO and high blood } \\
\text { eosinophils }(n=186)\end{array}$ & $p$-value \\
\hline \multicolumn{4}{|l|}{ Sex } \\
\hline n (\% non-missing) & $186(100.0)$ & $186(100.0)$ & \multirow[t]{2}{*}{1.0000} \\
\hline Male & 77 (41.4) & 77 (41.4) & \\
\hline \multicolumn{4}{|l|}{ Age } \\
\hline n (\% non-missing) & $186(100.0)$ & $186(100.0)$ & \multirow[t]{3}{*}{0.9834} \\
\hline Mean (SD) & $51.9(13.1)$ & $51.8(13.7)$ & \\
\hline Median (IQR) & $55.0(20.0)$ & $53.5(20.0)$ & \\
\hline \multicolumn{4}{|l|}{ Age group } \\
\hline n (\% non-missing) & $186(100.0)$ & $186(100.0)$ & \multirow[t]{4}{*}{0.1919} \\
\hline Under 35 & $22(11.8)$ & $24(12.9)$ & \\
\hline $35-65$ & $141(75.8)$ & $127(68.3)$ & \\
\hline $44-80$ & $23(12.4)$ & $35(18.8)$ & \\
\hline \multicolumn{4}{|l|}{ Smoking status } \\
\hline n (\% non-missing) & $186(100.0)$ & $186(100.0)$ & \multirow[t]{4}{*}{1.0000} \\
\hline Non-smoker & $67(36.0)$ & $67(36.0)$ & \\
\hline Ex-smoker & $22(11.8)$ & $22(11.8)$ & \\
\hline Current smoker & $67(36.0)$ & $67(36.0)$ & \\
\hline \multicolumn{4}{|l|}{ BMl } \\
\hline n (\% non-missing) & $184(98.4)$ & $184(98.4)$ & \multirow[t]{2}{*}{0.0492} \\
\hline Mean (SD) & $30.1(6.3)$ & $29.0(6.7)$ & \\
\hline \multicolumn{4}{|l|}{ FeNO } \\
\hline n (\% non-missing) & $186(100.0)$ & $186(100.0)$ & \multirow[t]{3}{*}{$<0.0001$} \\
\hline Mean (SD) & $16.5(7.8)$ & $28.9(23.8)$ & \\
\hline Median (IQR) & $16.0(12.0)$ & $23.0(22.0)$ & \\
\hline \multicolumn{4}{|c|}{ Blood eosinophil count } \\
\hline n (\% non-missing) & $186(100.0)$ & $184(98.9)$ & \multirow[t]{3}{*}{$<0.0001$} \\
\hline Mean (SD) & $0.2(0.1)$ & $0.4(0.2)$ & \\
\hline Median (IQR) & $0.2(0.1)$ & $0.4(0.2)$ & \\
\hline \multicolumn{4}{|c|}{ Active eczema diagnosis ${ }^{a}$} \\
\hline n (\% non-missing) & $186(100.0)$ & $186(100.0)$ & \multirow[t]{2}{*}{0.1876} \\
\hline Yes & $5(2.7)$ & $10(5.4)$ & \\
\hline \multicolumn{4}{|c|}{ Active rhinitis diagnosis $^{a}$} \\
\hline n (\% non-missing) & $186(100.0)$ & $186(100.0)$ & \multirow[t]{2}{*}{0.0720} \\
\hline Yes & $49(26.3)$ & $65(34.9)$ & \\
\hline \multicolumn{4}{|l|}{ Eczema diagnosis } \\
\hline n (\% non-missing) & $186(100.0)$ & $186(100.0)$ & \multirow[t]{2}{*}{0.2273} \\
\hline Yes & $57(30.6)$ & $68(36.6)$ & \\
\hline \multicolumn{4}{|l|}{ Rhinitis diagnosis } \\
\hline n (\% non-missing) & $186(100.0)$ & $186(100.0)$ & \multirow[t]{2}{*}{0.0272} \\
\hline Yes & $67(36.0)$ & $88(47.3)$ & \\
\hline IHD diagnosis & & & \\
\hline n (\% non-missing) & $186(100.0)$ & $186(100.0)$ & 0.4564 \\
\hline Yes & $7(3.8)$ & $10(5.4)$ & \\
\hline Heart failure diagnos & & & \\
\hline n (\% non-missing) & $186(100.0)$ & $186(100.0)$ & 0.3167 \\
\hline Yes & $0(0.0)$ & $1(0.5)$ & \\
\hline Hypertension diagno & & & \\
\hline n (\% non-missing) & $186(100.0)$ & $186(100.0)$ & 0.3212 \\
\hline Yes & $46(24.7)$ & $38(20.4)$ & \\
\hline
\end{tabular}


Table 3 (continued)

\begin{tabular}{|c|c|c|c|}
\hline Characteristics & $\begin{array}{l}\text { Non-high FeNO and non-high blood } \\
\text { eosinophils }(n=186)\end{array}$ & $\begin{array}{l}\text { Non-high FeNO and high blood } \\
\text { eosinophils }(n=186)\end{array}$ & p-value \\
\hline \multicolumn{4}{|l|}{ Diabetes diagnosis } \\
\hline n (\% non-missing) & $186(100.0)$ & $186(100.0)$ & \multirow[t]{2}{*}{0.5736} \\
\hline Yes & $14(7.5)$ & $17(9.1)$ & \\
\hline \multicolumn{4}{|l|}{ GERD active diagnosis } \\
\hline N (\% non-missing) & $186(100.0)$ & $186(100.0)$ & \multirow[t]{2}{*}{0.4352} \\
\hline Yes & $26(14.0)$ & $21(11.3)$ & \\
\hline \multicolumn{4}{|l|}{ Predicted peak flow } \\
\hline n (\% non-missing) & $98(52.7)$ & $101(54.3)$ & \multirow[t]{2}{*}{0.8525} \\
\hline Mean (SD) & $515.7(75.3)$ & $517.3(76.2)$ & \\
\hline \multicolumn{4}{|c|}{ ICS/LABA prescriptions per patient } \\
\hline n (\% non-missing) & $186(100.0)$ & $186(100.0)$ & \multirow[t]{3}{*}{0.0404} \\
\hline Mean (SD) & $3.8(3.9)$ & $4.4(3.8)$ & \\
\hline Median (IQR) & $3.0(6.0)$ & $4.0(5.0)$ & \\
\hline \multicolumn{4}{|c|}{ Mono ICS prescriptions per patient } \\
\hline n (\% non-missing) & $186(100.0)$ & $186(100.0)$ & \multirow[t]{3}{*}{0.0362} \\
\hline Mean (SD) & $1.3(2.5)$ & $0.7(1.6)$ & \\
\hline Median (IQR) & $0.0(1.0)$ & $0.0(1.0)$ & \\
\hline \multicolumn{4}{|c|}{ Mean daily SABA dosage $(\mu \mathrm{g})$} \\
\hline n (\% non-missing) & $186(100.0)$ & $186(100.0)$ & \multirow[t]{5}{*}{0.2585} \\
\hline$<100$ & $63(33.9)$ & $71(38.2)$ & \\
\hline $100-200$ & $57(30.6)$ & $48(25.8)$ & \\
\hline $201-400$ & $35(18.8)$ & $45(24.2)$ & \\
\hline$>400$ & $31(16.7)$ & $22(11.8)$ & \\
\hline \multicolumn{4}{|l|}{ ICS adherence ${ }^{b}$} \\
\hline n (\% non-missing) & $186(100.0)$ & $186(100.0)$ & \multirow[t]{3}{*}{0.8806} \\
\hline Mean (SD) & $69.2(55.2)$ & $66.2(53.3)$ & \\
\hline Median (IQR) & $54.8(71.2)$ & $56.1(57.5)$ & \\
\hline
\end{tabular}

All values in the table are $\mathrm{n}(\%)$ unless otherwise specified. High FeNO defined as $\geq 35 \mathrm{ppb}$; non-high FeNO $<35$ ppb; high blood eosinophil count defined as $\geq 0.300 \times 10^{9}$ cells $/ \mathrm{L}$; non-high blood eosinophil count $<0.300 \times 10^{9}$ cells $/ \mathrm{L}$

$B M I$ body mass index, FeNO fractional exhaled nitric oxide, GERD gastroesophageal reflux disease, ICS inhaled corticosteroid, IHD ischaemic heart disease, IQR interquartile range, $L A B A$ long-acting $\beta_{2}$-agonist, $p p b$ parts per billion, $S A B A$ short-acting $\beta_{2}$-agonist, $S D$ standard deviation

a Active denotes diagnosed in the year before FeNO reading or treated in the year before FeNO reading

b Medication Possession Ratio was calculated by dividing the total of 1 day's supply by the total number of days evaluated, multiplied by $100 \%$. The evaluation period for all patients was 365 days in the study year

The high FeNO cutoff of $\geq 35 \mathrm{ppb}$ has also been validated in several studies, in turn, identifying patients with uncontrolled asthma and a more severe asthma phenotype [31, 32]. These results suggest that a lower high FeNO cutoff of $\geq 35 \mathrm{ppb}$ instead of $\geq 50 \mathrm{ppb}$ (ATS criteria), on a background of raised blood eosinophil count, may still be relevant to predict those patients at significant risk of severe exacerbations. This implies that asthma patients with comparatively lower raised FeNO concentrations and elevated blood eosinophil count may require further treatment, suggesting that the risk of severe exacerbations may potentially be over and above that provided by a traditional severity-based classification.
Few studies have evaluated the predictive value of the combination of blood eosinophil count and FeNO concentration in asthma. However, available studies have demonstrated that combining FeNO and blood eosinophil count has an additive effect in predicting wheeze, frequent exacerbations, impaired lung function, and bronchial hyper-responsiveness [23, 38]. The National Institute for Health and Care Excellence [39] and the British Thoracic Society recommend FeNO measurement to guide diagnosis and treatment of eosinophilic asthma [40]. Use of FeNO as a diagnostic tool is increasing. In UK primary care practices, FeNO monitoring is also being used to guide decisions on ICS usage or stepup therapy [37]. In addition, the 2019 Global Initiative 
Table 4 Categorisation 2: non-high FeNO and non-high blood eosinophils vs. high FeNO and non-high blood eosinophils

\begin{tabular}{|c|c|c|c|}
\hline Characteristics & $\begin{array}{l}\text { Non-high FeNO and non-high blood } \\
\text { eosinophils }(n=98)\end{array}$ & $\begin{array}{l}\text { High FeNO and non-high blood } \\
\text { eosinophils }(n=98)\end{array}$ & $p$-value \\
\hline \multicolumn{4}{|l|}{ Sex } \\
\hline n (\% non-missing) & $98(100.0)$ & $98(100.0)$ & \multirow[t]{2}{*}{1.0000} \\
\hline Male & $41(41.8)$ & $41(41.8)$ & \\
\hline \multicolumn{4}{|l|}{ Age } \\
\hline n (\% non-missing) & $98(100.0)$ & $98(100.0)$ & \multirow[t]{3}{*}{1.0000} \\
\hline Mean (SD) & $48.8(15.3)$ & $48.6(15.6)$ & \\
\hline Median (IQR) & $53.0(27.0)$ & $53.0(27.0)$ & \\
\hline \multicolumn{4}{|l|}{ Age group } \\
\hline n (\% non-missing) & $98(100.0)$ & $98(100.0)$ & \multirow[t]{4}{*}{0.3072} \\
\hline Under 35 & $23(23.5)$ & $24(24.5)$ & \\
\hline $35-65$ & $65(66.3)$ & $57(58.2)$ & \\
\hline $66-80$ & $10(10.2)$ & $17(17.3)$ & \\
\hline \multicolumn{4}{|l|}{ Smoking status } \\
\hline n (\% non-missing) & $98(100.0)$ & $98(100.0)$ & \multirow[t]{4}{*}{1.0000} \\
\hline Non-smoker & $53(54.1)$ & $53(54.1)$ & \\
\hline Ex-smoker & $8(8.2)$ & $8(8.2)$ & \\
\hline Current smoker & $23(23.5)$ & $23(23.5)$ & \\
\hline \multicolumn{4}{|l|}{ BMl } \\
\hline n (\% non-missing) & $96(98.0)$ & 94 (95.9) & \multirow[t]{3}{*}{0.0063} \\
\hline Mean (SD) & $29.3(6.2)$ & $26.9(5.8)$ & \\
\hline Median (IQR) & $27.9(8.6)$ & $25.7(7.6)$ & \\
\hline \multicolumn{4}{|l|}{ FeNO } \\
\hline n (\% non-missing) & $98(100.0)$ & $98(100.0)$ & \multirow[t]{3}{*}{$<0.000$} \\
\hline Mean (SD) & $17.7(7.9)$ & $60.0(31.8)$ & \\
\hline Median (IQR) & $17.0(10.0)$ & $50.0(25.0)$ & \\
\hline \multicolumn{4}{|c|}{ Blood eosinophil count } \\
\hline n (\% non-missing) & $98(100.0)$ & $97(99.0)$ & \multirow[t]{3}{*}{$<0.0001$} \\
\hline Mean (SD) & $0.2(0.1)$ & $0.3(0.3)$ & \\
\hline Median (IQR) & $0.2(0.1)$ & $0.3(0.3)$ & \\
\hline \multicolumn{4}{|c|}{ Active eczema diagnosis ${ }^{\mathrm{a}}$} \\
\hline n (\% non-missing) & $98(100.0)$ & $98(100.0)$ & \multirow[t]{2}{*}{1.000} \\
\hline Yes & $4(4.1)$ & $4(4.1)$ & \\
\hline \multicolumn{4}{|c|}{ Active rhinitis diagnosis ${ }^{\mathrm{a}}$} \\
\hline n (\% non-missing) & $98(100.0)$ & $98(100.0)$ & \multirow[t]{2}{*}{0.749} \\
\hline Yes & $26(26.5)$ & $28(28.6)$ & \\
\hline \multicolumn{4}{|l|}{ Eczema diagnosis } \\
\hline n (\% non-missing) & $98(100.0)$ & $98(100.0)$ & \multirow[t]{2}{*}{0.8763} \\
\hline Yes & $29(29.6)$ & $30(30.6)$ & \\
\hline \multicolumn{4}{|l|}{ Rhinitis diagnosis } \\
\hline n (\% non-missing) & $98(100.0)$ & $98(100.0)$ & \multirow[t]{2}{*}{0.4546} \\
\hline Yes & $32(32.7)$ & $37(37.8)$ & \\
\hline IHD diagnosis & & & \\
\hline n (\% non-missing) & $98(100.0)$ & $98(100.0)$ & 0.7003 \\
\hline Yes & $4(4.1)$ & $3(3.1)$ & \\
\hline Heart failure diagnos & & & \\
\hline n (\% non-missing) & $98(100.0)$ & $98(100.0)$ & \\
\hline Yes & $0(0.0)$ & $0(0.0)$ & \\
\hline Hypertension diagno & & & \\
\hline n (\% non-missing) & $98(100.0)$ & $98(100.0)$ & 0.0967 \\
\hline
\end{tabular}


Table 4 (continued)

\begin{tabular}{|c|c|c|c|}
\hline Characteristics & $\begin{array}{l}\text { Non-high FeNO and non-high blood } \\
\text { eosinophils }(n=98)\end{array}$ & $\begin{array}{l}\text { High FeNO and non-high blood } \\
\text { eosinophils }(n=98)\end{array}$ & $p$-value \\
\hline Yes & $29(29.6)$ & $19(19.4)$ & \\
\hline \multicolumn{4}{|l|}{ Diabetes diagnosis } \\
\hline n (\% non-missing) & $98(100.0)$ & $98(100.0)$ & 0.5513 \\
\hline Yes & $7(7.1)$ & $5(5.1)$ & \\
\hline \multicolumn{4}{|l|}{ GERD active diagnosis } \\
\hline n (\% non-missing) & $98(100.0)$ & $98(100.0)$ & 0.6018 \\
\hline Yes & $9(9.2)$ & $7(7.1)$ & \\
\hline \multicolumn{4}{|l|}{ Predicted peak flow } \\
\hline n (\% non-missing) & $55(56.1)$ & $71(72.4)$ & 0.6615 \\
\hline Mean (SD) & $520.7(67.4)$ & $515.9(68.6)$ & \\
\hline Median (IQR) & $490.8(137.4)$ & $493.7(125.0)$ & \\
\hline \multicolumn{4}{|c|}{ ICS/LABA prescriptions per patient } \\
\hline n (\% non-missing) & $98(100.0)$ & $98(100.0)$ & 0.1318 \\
\hline Mean (SD) & $2.6(3.1)$ & $3.4(3.7)$ & \\
\hline Median (IQR) & $1.0(4.0)$ & $2.0(5.0)$ & \\
\hline \multicolumn{4}{|c|}{ Mono ICS prescriptions per patient } \\
\hline n (\% non-missing) & $98(100.0)$ & $98(100.0)$ & 0.0295 \\
\hline Mean (SD) & $1.6(2.8)$ & $0.9(1.9)$ & \\
\hline Median (IQR) & $0.0(2.0)$ & $0.0(1.0)$ & \\
\hline \multicolumn{4}{|c|}{ Mean daily SABA dosage $(\mu \mathrm{g})$} \\
\hline n (\% non-missing) & $98(100.0)$ & $98(100.0)$ & 0.3731 \\
\hline$<100$ & $31(31.6)$ & $40(40.8)$ & \\
\hline $100-200$ & $32(32.7)$ & $22(22.4)$ & \\
\hline $201-400$ & $20(20.4)$ & $22(22.4)$ & \\
\hline$>400$ & $15(15.3)$ & $14(14.3)$ & \\
\hline \multicolumn{4}{|l|}{ ICS adherence ${ }^{b}$} \\
\hline n (\% non-missing) & $98(100.0)$ & $98(100.0)$ & 0.4778 \\
\hline Mean (SD) & $58.1(43.5)$ & $65.7(67.6)$ & \\
\hline Median (IQR) & $49.3(57.5)$ & $49.3(54.8)$ & \\
\hline
\end{tabular}

All values in the table are $\mathrm{n}(\%)$ unless otherwise specified. High FeNO defined as $\geq 35$ ppb; non-high FeNO $<35$ ppb; high blood eosinophil count defined as $\geq 0.300 \times 10^{9}$ cells/L; non-high blood eosinophil count $<0.300 \times 10^{9}$ cells $/ \mathrm{L}$

$B M I$ body mass index, FeNO fractional exhaled nitric oxide, GERD gastroesophageal reflux disease, ICS inhaled corticosteroid, IHD ischaemic heart disease, IQR interquartile range, $L A B A$ long-acting $\beta_{2}$-agonist, $p p b$ parts per billion, $S A B A$ short-acting $\beta_{2}$-agonist, $S D$ standard deviation

a Active denotes diagnosed in the year before FeNO reading or treated in the year before FeNO reading

b Medication Possession Ratio was calculated by dividing the total of 1 day's supply by the total number of days evaluated, multiplied by $100 \%$. The evaluation period for all patients was 365 days in the study year

for Asthma strategy report [14] recommends the use of FeNO and/or blood eosinophil counts to determine asthma phenotype and for biomarker-guided selection of biologics. Thus, composite, non-invasive biomarkers, such as FeNO and easily obtainable blood eosinophil count, may provide insight into a patient's risk of exacerbations as well as guide asthma treatment.

Other well-characterised risk factors for asthma exacerbations include prior exacerbations, OCS use, and underlying lung function impairment $[41,42]$. The combination of these standard medical history/lung functionbased assessments and objective biomarkers, such as
FeNO and blood eosinophil count, may improve the prediction of asthma exacerbations. Furthermore, within the limits of the data, our results indicate that the prognostic value of both FeNO and blood eosinophil count as complementary biomarkers appears to be greater than that provided by these traditional clinical assessments [41, 42].

This study has several limitations. The power analysis performed at the protocol stage demonstrated that more patients were required for sufficient power to demonstrate a difference between four groups than were available. Secondly, the OPCRD data set comprised information 
Table 5 Categorisation 2: non-high FeNO and non-high blood eosinophils vs. high FeNO and high blood eosinophils

\begin{tabular}{|c|c|c|c|}
\hline Characteristics & $\begin{array}{l}\text { Non-high FeNO and non-high blood } \\
\text { eosinophils }(n=53)\end{array}$ & $\begin{array}{l}\text { High FeNO and high blood eosinophils } \\
(n=53)\end{array}$ & p-value \\
\hline \multicolumn{4}{|l|}{ Sex } \\
\hline n (\% non-missing) & $53(100.0)$ & $53(100.0)$ & \multirow[t]{2}{*}{1.0000} \\
\hline Male & $48.4(16.7)$ & $48.2(16.9)$ & \\
\hline \multicolumn{4}{|l|}{ Age } \\
\hline n (\% non-missing) & $53.0(100.0)$ & $53.0(100.0)$ & \multirow[t]{3}{*}{0.964} \\
\hline Mean (SD) & $48.4(16.7)$ & $48.2(16.9)$ & \\
\hline Median (IQR) & $53.0(32.0)$ & $53.0(31.0)$ & \\
\hline \multicolumn{4}{|l|}{ Age group } \\
\hline n (\% non-missing) & $53(100.0)$ & $53(100.0)$ & \multirow[t]{4}{*}{0.8655} \\
\hline Under 35 & $14(26.4)$ & $14(26.4)$ & \\
\hline $35-65$ & $31(58.5)$ & $29(54.7)$ & \\
\hline $66-80$ & $8(15.1)$ & $10(18.9)$ & \\
\hline \multicolumn{4}{|l|}{ Smoking status } \\
\hline n (\% non-missing) & $46(86.8)$ & $46(86.8)$ & \multirow[t]{4}{*}{1.0000} \\
\hline Non-smoker & $31(58.5)$ & $31(58.5)$ & \\
\hline Ex-smoker & $4(7.5)$ & $4(7.5)$ & \\
\hline Current smoker & $11(20.8)$ & $11(20.8)$ & \\
\hline \multicolumn{4}{|l|}{ BMI } \\
\hline n (\% non-missing) & $52(98.1)$ & $51(96.2)$ & \multirow[t]{3}{*}{0.0386} \\
\hline Mean (SD) & $29.0(5.9)$ & $26.8(5.6)$ & \\
\hline Median (IQR) & $28.4(8.3)$ & $25.6(7.1)$ & \\
\hline \multicolumn{4}{|l|}{ FeNO } \\
\hline n (\% non-missing) & $53(100.0)$ & $53(100.0)$ & \multirow[t]{3}{*}{$<0.0001$} \\
\hline Mean (SD) & $18.6(7.7)$ & $57.8(26.4)$ & \\
\hline Median (IQR) & $20.0(10.0)$ & $49.0(28.0)$ & \\
\hline \multicolumn{4}{|c|}{ Blood eosinophil count } \\
\hline n (\% non-missing) & $53(100.0)$ & $52(98.1)$ & \multirow[t]{3}{*}{$<0.0001$} \\
\hline Mean (SD) & $0.1(0.1)$ & $0.5(0.3)$ & \\
\hline Median (IQR) & $0.1(0.1)$ & $0.5(0.2)$ & \\
\hline \multicolumn{4}{|c|}{ Active eczema diagnosis $^{a}$} \\
\hline n (\% non-missing) & $53(100.0)$ & $53(100.0)$ & \multirow[t]{2}{*}{1.0000} \\
\hline Yes & $2(3.8)$ & $2(3.8)$ & \\
\hline \multicolumn{4}{|c|}{ Active rhinitis diagnosis ${ }^{a}$} \\
\hline n (\% non-missing) & $53(100.0)$ & $53(100.0)$ & \multirow[t]{2}{*}{0.4052} \\
\hline Yes & $15(28.3)$ & $19(35.8)$ & \\
\hline \multicolumn{4}{|l|}{ Eczema diagnosis } \\
\hline n (\% non-missing) & $53(100.0)$ & $53(100.0)$ & \multirow[t]{2}{*}{0.5355} \\
\hline Yes & $16(30.2)$ & $19(35.8)$ & \\
\hline \multicolumn{4}{|l|}{ Rhinitis diagnosis } \\
\hline n (\% non-missing) & $53(100.0)$ & $53(100.0)$ & \multirow[t]{2}{*}{0.0451} \\
\hline Yes & $15(28.3)$ & $25(47.2)$ & \\
\hline IHD diagnosis & & & \\
\hline n (\% non-missing) & $53(100.0)$ & $53(100.0)$ & 0.5581 \\
\hline Yes & $1(1.9)$ & $2(3.8)$ & \\
\hline Heart failure diagnos & & & \\
\hline n (\% non-missing) & $53(100.0)$ & $53(100.0)$ & \\
\hline Yes & $0(0.0)$ & $0(0.0)$ & \\
\hline Hypertension diagno & & & \\
\hline n (\% non-missing) & $53(100.0)$ & $53(100.0)$ & 0.0990 \\
\hline
\end{tabular}


Table 5 (continued)

\begin{tabular}{|c|c|c|c|}
\hline Characteristics & $\begin{array}{l}\text { Non-high FeNO and non-high blood } \\
\text { eosinophils }(n=53)\end{array}$ & $\begin{array}{l}\text { High FeNO and high blood eosinophils } \\
(n=53)\end{array}$ & p-value \\
\hline Yes & $15(28.3)$ & $8(15.1)$ & \\
\hline \multicolumn{4}{|l|}{ Diabetes diagnosis } \\
\hline n (\% non-missing) & $53(100.0)$ & $53(100.0)$ & 1.0000 \\
\hline Yes & $3(5.7)$ & $3(5.7)$ & \\
\hline \multicolumn{4}{|l|}{ GERD active diagnosis } \\
\hline n (\% non-missing) & $53(100.0)$ & $53(100.0)$ & 0.5063 \\
\hline Yes & $6(11.3)$ & $4(7.5)$ & \\
\hline \multicolumn{4}{|l|}{ Predicted peak flow } \\
\hline n (\% non-missing) & $35(66.0)$ & $39(73.6)$ & 0.8838 \\
\hline Mean (SD) & $513.0(66.7)$ & $510.3(69.9)$ & \\
\hline Median (IQR) & $487.5(122.0)$ & $481.2(128.0)$ & \\
\hline \multicolumn{4}{|c|}{ ICS/LABA prescriptions per patient } \\
\hline n (\% non-missing) & $53(100.0)$ & $53(100.0)$ & 0.2204 \\
\hline Mean (SD) & $3.3(3.9)$ & $4.0(4.0)$ & \\
\hline Median (IQR) & $2.0(5.0)$ & $3.0(5.0)$ & \\
\hline \multicolumn{4}{|c|}{ Mono ICS prescriptions per patient } \\
\hline n (\% non-missing) & $53(100.0)$ & $53(100.0)$ & 0.1944 \\
\hline Mean (SD) & $1.3(2.3)$ & $0.8(1.7)$ & \\
\hline Median (IQR) & $0.0(1.0)$ & $0.0(1.0)$ & \\
\hline \multicolumn{4}{|c|}{ Mean daily SABA dosage $(\mu \mathrm{g})$} \\
\hline n (\% non-missing) & $53(100.0)$ & $53(100.0)$ & 0.6923 \\
\hline$<100$ & $15(28.3)$ & $16(30.2)$ & \\
\hline $100-200$ & $19(35.8)$ & $14(26.4)$ & \\
\hline $201-400$ & $10(18.9)$ & $14(26.4)$ & \\
\hline$>400$ & $9(17.0)$ & $9(17.0)$ & \\
\hline \multicolumn{4}{|l|}{ ICS adherence ${ }^{b}$} \\
\hline n (\% non-missing) & $53(100.0)$ & $53(100.0)$ & 0.8149 \\
\hline Mean (SD) & $64.1(45.3)$ & $68.6(72.5)$ & \\
\hline Median (IQR) & $54.8(54.8)$ & $49.3(49.3)$ & \\
\hline
\end{tabular}

All values in the table are $\mathrm{n}(\%)$ unless otherwise specified. High FeNO defined as $\geq 35 \mathrm{ppb}$; non-high FeNO $<35$ ppb; high blood eosinophil count defined as $\geq 0.300 \times 10^{9}$ cells/L; non-high blood eosinophil count $<0.300 \times 10^{9}$ cells $/ \mathrm{L}$

$B M I$ body mass index, FeNO fractional exhaled nitric oxide, GERD gastroesophageal reflux disease, ICS inhaled corticosteroid, IHD ischaemic heart disease, IQR interquartile range, $L A B A$ long-acting $\beta_{2}$-agonist, $p p b$ parts per billion, $S A B A$ short-acting $\beta_{2}$-agonist, $S D$ standard deviation

a Active denotes diagnosed in the year before FeNO reading or treated in the year before FeNO reading

b Medication Possession Ratio was calculated by dividing the total of 1 day's supply by the total number of days evaluated, multiplied by $100 \%$. The evaluation period for all patients was 365 days in the study year

collected for clinical and routine use rather than specifically for research purposes. Although extensive quality control and validity checks were conducted at the practice level, the validity and completeness of individual patient records can be limited. Since blood eosinophil measurements and FeNO readings are not collected routinely, patients with asthma who had both blood eosinophil counts and FeNO measured may not have been representative of the overall asthma population. In addition, the time from when the blood eosinophil count reading was taken to the index date varied considerably. Although high blood eosinophil counts have been observed to be a stable phenotype, at least during a 1-year period [11], further studies are required to investigate the potential long-term stability of blood eosinophil counts. As with all observational studies, confounding variables, arising from systematic differences between the patients being compared, may have complicated interpretation of these results. In this study, confounding was minimised by fitting multivariate models that adjusted patient characteristics that may have varied between patient groups. However, despite these measures, confounding by unmeasured variables may have been present. Finally, adherence to ICS was not a prerequisite to enter the study, and as a result adherence was not 


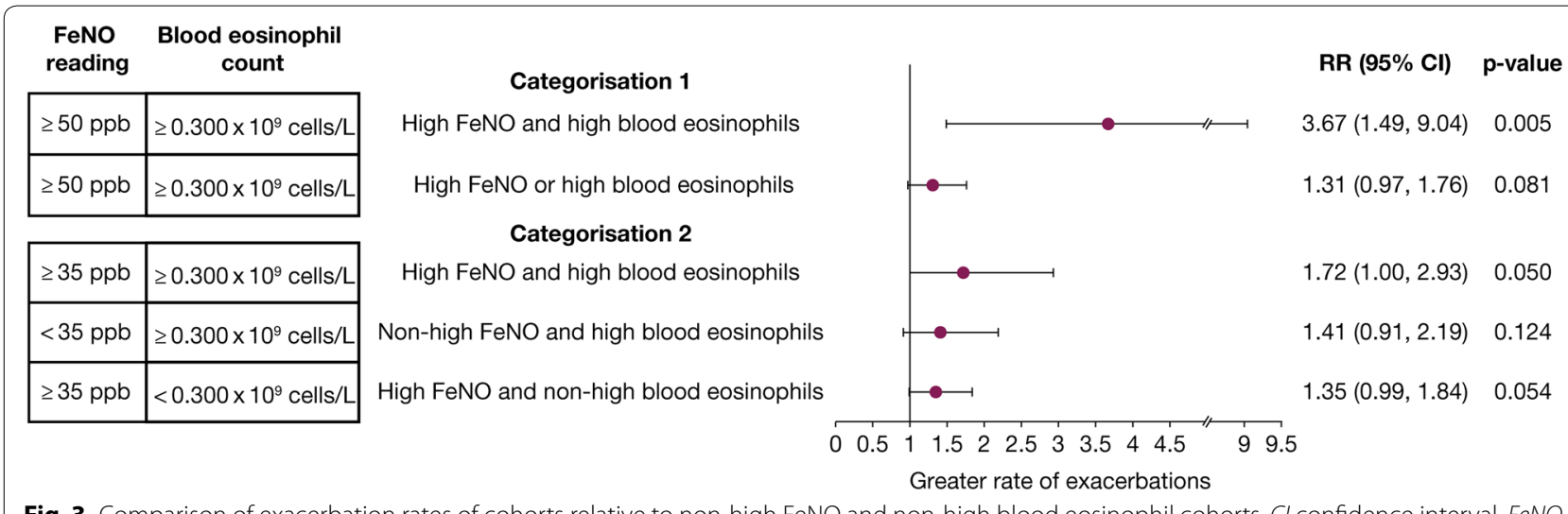

Fig. 3 Comparison of exacerbation rates of cohorts relative to non-high FeNO and non-high blood eosinophil cohorts. Cl confidence interval, FeNO fractional exhaled nitric oxide, $R R$ rate ratio

optimal. While ICS adherence between each cohort and reference group was not significantly different, it is likely that FeNO concentrations and blood eosinophil counts may be differentially predictive in patients receiving or not receiving their prescribed ICS medications.

Results of this study need to be confirmed in a prospective study in a larger patient population before high FeNO concentrations and high blood eosinophil counts can be advocated as a composite biomarker. Notably, patients with elevated FeNO concentration on a background of high blood eosinophil counts represent a potentially high-risk group of patients. Such severe asthma patients will benefit from studies conducted in larger epidemiological cohorts in primary care settings, as well as in severe asthma cohorts, such as the International Severe Asthma Registry [43], a global registry of adult patients with severe asthma, and the CHRONICLE study [44], an ongoing non-interventional, prospective cohort study of adults with severe asthma treated by specialists in the United States. Overall, findings from this study, based on real-life data obtained from a validated database, warrant further investigation into the role of FeNO and blood eosinophils as biomarkers in the treatment and management of asthma.

\section{Conclusions}

The combination of raised FeNO concentrations and raised blood eosinophil counts was associated with a greater exacerbation rate compared with neither biomarker raised in the year preceding the FeNO reading. FeNO concentration and blood eosinophil count are simple measurements that could, together, improve the identification of patients with asthma in primary and secondary care at risk of exacerbations, and thus, guide additional considerations in the treatment of their asthma.

\section{Additional file}

Additional file 1. Statistically significant differences between unmatched patient groups for categorisation 1 and 2 and frequency of exacerbations between matched biomarker groups.

\section{Abbreviations}

ADEPT: Anonymised Data Ethics and Protocol Transparency; ATS: American Thoracic Society; BEC: blood eosinophil count; BMI: body mass index; Cl: confidence interval; FeNO: fractional exhaled nitric oxide; ICS: inhaled corticosteroid; OCS: oral corticosteroids; OPCRD: optimum patient care research database; RR: rate ratio; SD: standard deviation.

\section{Acknowledgements}

The authors thank Gokul Gopalan and Sadia Halim for their contributions to the development of a poster based on the findings of this study, which was presented at the British Thoracic Society (BTS) Winter Meeting, 6-8 December 2017, London, United Kingdom. Editorial support was provided by Michelle Rebello, PhD, of Cactus Communications (Mumbai, India) and Michael A. Nissen, ELS, of AstraZeneca (Gaithersburg, MD, USA) in accordance with Good Publication Practice (GPP3) guidelines (http://www.ismpp.org/gpp3). This support was fully funded by AstraZeneca.

\section{Authors' contributions}

All authors contributed equally to the design of the study, data analysis and drafting, and revising the manuscript. All authors read and approved the final manuscript.

Funding

This study was funded by AstraZeneca.

\section{Availability of data and materials}

Data underlying the findings described in this manuscript may be obtained in accordance with AstraZeneca's data sharing policy described at https://astra zenecagrouptrials.pharmacm.com/ST/Submission/Disclosure.

\section{Ethics approval and consent to participate}

The study was registered under the established study database, namely, the European Network of Centres for Pharmacoepidemiology and Pharmacovigilance under the Registration Number EUPAS16891. Ethical approvals were obtained from the Anonymised Data Ethics \& Protocol Transparency committee (ADEPT1017).

Consent for publication

Not applicable. 


\section{Competing interests}

DP reports grants and/or personal fees from Aerocrine, AKL Research and Development Ltd., Almirall, Amgen, AstraZeneca, Boehringer Ingelheim, the British Lung Foundation, Chiesi, Cipla, GlaxoSmithKline, Kyorin, Merck, Mylan, Mundipharma, Napp, Novartis, Pfizer, Regeneron Pharmaceuticals, the Respiratory Effectiveness Group, Sanofi Genzyme, Skyepharma, Teva, Theravance, the UK National Health Service, Zentiva (Sanofi Generics); non-financial support from Efficacy and Mechanism Evaluation Programme and Health Technology Assessment, outside the submitted work; stock/stock options from AKL Research and Development Ltd.; and owns 74\% of the social enterprise Optimum Patient Care Ltd. (Australia and UK) and 74\% of Observational and Pragmatic Research Institute Pte Ltd. (Singapore). SB reports grants and personal fees from TEVA, personal fees from Boehringer Ingelheim,

AstraZeneca, Sanofi, and Mylan, outside the submitted work. IP has received speaker's honoraria for speaking at sponsored meetings from AstraZeneca, Boehringer Ingelheim, Aerocrine, Almirall, Novartis, Teva, Chiesi, and GlaxoSmithKline, and payments for organising educational events from AstraZeneca and Teva. He has received honoraria for attending advisory panels with Genentech, Regeneron, AstraZeneca, Boehringer Ingelheim, GlaxoSmithKline, Novartis, Teva, Merck, Sanofi, Circassia, Chiesi, and Knopp. He has received sponsorship to attend international scientific meetings from Boehringer Ingelheim, GlaxoSmithKline, AstraZeneca, Teva, and Chiesi. He has received a grant from Chiesi to support a phase 2 clinical trial in Oxford. NR reports grants and personal fees from Boehringer Ingelheim, Novartis, Pfizer and personal fees from Teva, GlaxoSmithKline, AstraZeneca, Chiesi, Mundipharma, Cipla, Sanofi, Sandoz, 3 M, and Zambon. DH reports personal fees and/or non-financial support from AstraZeneca, Boehringer Ingelheim, Chiesi, GlaxoSmithKline, Novartis, and Pfizer, outside the submitted work. LB has nothing to disclose. OU has received grants and/or personal fees from AstraZeneca, Boehringer Ingelheim, Chiesi, Aerocrine, GlaxoSmithKline, Napp, Mundipharma, Sandoz, Prosonix, Takeda, Zentiva, Edmond Pharma, Cipla, and Pearl Therapeutics. GB reports personal fees from AstraZeneca, Boehringer Ingelheim, Chiesi, GlaxoSmithKline, Novartis, Sanofi, and Teva, outside the submitted work. SWM reports other from Observational and Pragmatic Research Institute Pte Ltd. (OPRI), outside the submitted work. He was employed by the OPRI at the time the analyses were conducted; OPRI has conducted paid research in respiratory disease on behalf of the following organisations in the past 5 years: Anaxys, AstraZeneca, Boehringer Ingelheim, British Lung Foundation, Chiesi, Circassia (formerly Aerocrine), GlaxoSmithKline, Harvey Walsh, Mapi, Morningside Healthcare, Mundipharma, Mylan (formerly Meda), Napp, Novartis, Orion, Plymouth University, Regeneron, Respiratory Effectiveness Group, Roche, Sanofi, Takeda, Teva, University of East Anglia, and Zentiva (a Sanofi company). SR was an employee of AstraZeneca at the time the analyses were conducted.

\section{Author details}

${ }^{1}$ Observational and Pragmatic Research Institute, Singapore, Singapore. 2 Academic Primary Care, Division of Applied Health Sciences, University of Aberdeen, Polwarth Building, Foresterhill, Aberdeen AB25 2ZD, UK. ${ }^{3}$ Woolcock Institute of Medical Research, University of Sydney, Sydney, Australia. ${ }^{4}$ Oxford NIHR Respiratory Biomedical Research Centre, Nuffield Department of Medicine, University of Oxford, Oxford, UK. ${ }^{5}$ University Paris Descartes, Paris, France. ${ }^{6}$ Royal Devon and Exeter Hospital, Exeter, UK. ${ }^{7}$ Lund University, Lund, Sweden. ${ }^{8}$ Imperial College London, London, UK. ${ }^{9}$ Ghent University Hospital, Ghent, Belgium. ${ }^{10}$ Global Medical Affairs, AstraZeneca, Gaithersburg, USA

Received: 12 June 2019 Accepted: 8 August 2019

Published online: 21 August 2019

\section{References}

1. Vos T, Abajobir AA, Abate KH, Abbafati C, Abbas KM, Abd-Allah F, et al. Global, regional, and national incidence, prevalence, and years lived with disability for 328 diseases and injuries for 195 countries, 1990-2016: a systematic analysis for the Global Burden of Disease Study 2016. Lancet. 2017;390:1211-59.

2. Naghavi M, Abajobir AA, Abbafati C, Abbas KM, Abd-Allah F, Abera SF, et al. Global, regional, and national age-sex specific mortality for 264 causes of death, 1980-2016: a systematic analysis for the Global Burden of Disease Study 2016. Lancet. 2017;390:1151-210.
3. Bahadori K, Doyle-Waters MM, Marra C, Lynd L, Alasaly K, Swiston J, et al. Economic burden of asthma: a systematic review. BMC Pul Med. 2009;9:24.

4. Wenzel SE. Asthma phenotypes: the evolution from clinical to molecular approaches. Nat Med. 2012;18:716-25.

5. Zhang JY, Wenzel SE. Tissue and BAL based biomarkers in asthma. Immunol Allergy Clin North Am. 2007;27:623-32.

6. Douwes J, Gibson P, Pekkanen J, Pearce N. Non-eosinophilic asthma: importance and possible mechanisms. Thorax. 2002;57:643-8.

7. Jatakanon A, Lim S, Barnes PJ. Changes in sputum eosinophils predict loss of asthma control. Am J Respir Crit Care Med. 2000;161:64-72.

8. Green RH, Brightling CE, McKenna S, Hargadon B, Parker D, Bradding P, et al. Asthma exacerbations and sputum eosinophil counts: a randomised controlled trial. Lancet. 2002;360:1715-21.

9. Reddel HK, Taylor DR, Bateman ED, Boulet LP, Boushey HA, Busse WW, et al. An official American Thoracic Society/European Respiratory Society statement: asthma control and exacerbations: standardizing endpoints for clinical asthma trials and clinical practice. Am J Respir Crit Care. 2009;180:59-99.

10. Chung KF, Wenzel SE, Brozek JL, Bush A, Castro M, Sterk PJ, et al. International ERS/ATS guidelines on definition, evaluation and treatment of severe asthma. Eur Respir J. 2014:43:343-73.

11. Price DB, Rigazio A, Campbell JD, Bleecker ER, Corrigan CJ, Thomas M, et al. Blood eosinophil count and prospective annual asthma disease burden: a UK cohort study. Lancet Respir Med. 2015;3:849-58.

12. Tran TN, Khatry DB, Ke X, Ward CK, Gossage D. High blood eosinophil count is associated with more frequent asthma attacks in asthma patients. Ann Allergy Asthma Immunol. 2014;113:19-24.

13. Kerkhof M, Tran TN, van den Berge M, Brusselle GG, Gopalan G, Jones RCM, et al. Association between blood eosinophil count and risk of readmission for patients with asthma: historical cohort study. PLoS ONE. 2018;13:e0201143.

14. Global Initiative for Asthma. Global strategy for asthma management and prevention. 2019. https://ginasthma.org/wp-content/uploads/2019/06/ GINA-2019-main-report-June-2019-wms.pdf .(Accessed 18 July 2019).

15. Bleecker ER, FitzGerald JM, Chanez P, Papi A, Weinstein SF, Barker P, et al. Efficacy and safety of benralizumab for patients with severe asthma uncontrolled with high-dosage inhaled corticosteroids and long-acting $\beta 2$-agonists (SIROCCO): a randomised, multicentre, placebo-controlled phase 3 trial. Lancet. 2016:388:2115-27.

16. Wenzel S, Ford L, Pearlman D, Spector S, Sher L, Skobieranda F, et al. Dupilumab in persistent asthma with elevated eosinophil levels. N Engl J Med. 2013;368:2455-66.

17. Pavord ID, Korn S, Howarth P, Bleecker ER, Buhl R, Keene ON, et al. Mepolizumab for severe eosinophilic asthma (DREAM): a multicentre, double-blind, placebo-controlled trial. Lancet. 2012;380:651-9.

18. Castro M, Zangrilli J, Wechsler ME, Bateman ED, Brusselle GG, Bardin P, et al. Reslizumab for inadequately controlled asthma with elevated blood eosinophil counts: results from two multicentre, parallel, double-blind, randomised, placebo-controlled, phase 3 trials. Lancet Respir Med. 2015:3:355-66

19. Dweik RA, Boggs PB, Erzurum SC, Irvin CG, Leigh MW, Lundberg JO, et al. An official ATS clinical practice guideline: interpretation of exhaled nitric oxide levels (FENO) for clinical applications. Am J Respir Crit Care Med. 2011;184:602-15.

20. Price DB, Buhl R, Chan A, Freeman D, Gardener E, Godley C, et al. Fractional exhaled nitric oxide as a predictor of response to inhaled corticosteroids in patients with non-specific respiratory symptoms and insignificant bronchodilator reversibility: a randomised controlled trial. Lancet Respir Med. 2018;6:29-39.

21. Gelb AF, Flynn Taylor C, Shinar CM, Gutierrez C, Zamel N, et al. Role of spirometry and exhaled nitric oxide to predict exacerbations in treated asthmatics. Chest. 2006;129:1492-9.

22. Zeiger RS, Schatz M, Zhang F, Crawford WW, Kaplan MS, Roth RM, et al. Elevated exhaled nitric oxide is a clinical indicator of future uncontrolled asthma in asthmatic patients on inhaled corticosteroids. J Allergy Clin Immunol. 2011;128:412-4.

23. Malinovschi A, Fonseca JA, Jacinto T, Alving K, Janson C. Exhaled nitric oxide levels and blood eosinophil counts independently associate with wheeze and asthma events in National Health and Nutrition Examination Survey subjects. J Allergy Clin Immunol. 2013;132(821-27):e5. 
24. Anderson WJ, Short PM, Williamson PA, Manoharan A, Lipworth BJ, et al. The inverse agonist propranolol confers no corticosteroid-sparing activity in mild-to-moderate persistent asthma. Clin Sci (Lond). 2014;127:635-43.

25. Wilson AM, Lipworth BJ. Dose-response evaluation of the therapeutic index for inhaled budesonide in patients with mild-to-moderate asthma. Am J Med. 2000;108:269-75.

26. Strunk RC, Szefler SJ, Phillips BR, Zeiger RS, Chinchilli VM, Larsen G, et al. Relationship of exhaled nitric oxide to clinical and inflammatory markers of persistent asthma in children. J Allergy Clin Immunol. 2003;112:883-92.

27. Corren J, Lemanske RF, Hanania NA, Korenblat PE, Parsey MV, Arron JR, et al. Lebrikizumab treatment in adults with asthma. N Engl J Med. 2011;365:1088-98.

28. Haldar P, Brightling CE, Hargadon B, Gupta S, Monteiro W, Sousa A, et al. Mepolizumab and exacerbations of refractory eosinophilic asthma. $\mathrm{N}$ Engl J Med. 2009;360:973-84.

29. Optimum Patient Care. The Optimum Patient Care Research Database (OPCRD). 2017. http://optimumpatientcare.org/database-overview/. Accessed 12 June 2019.

30. Optimum Patient Care. Anonymised Data Ethics \& Protocol Transparency (ADEPT) committee. http://optimumpatientcare.org/adept-commitee/. Accessed 12 June 2019.

31. Kerkhof M, Freeman D, Jones R, Chisholm A, Price DB. Predicting frequent COPD exacerbations using primary care data. Int J Chron Obstruct Pulmon Dis. 2015;10:2439-50.

32. Blakey JD, Price DB, Pizzichini E, Popov TA, Dimitrov BD, Postma DS, et al. Identifying risk of future asthma attacks using UK medical record data: a respiratory effectiveness group initiative. J Allergy Clin Immunol Pract. 2017;5:1015-24.

33. Calhoun WJ, Ameredes BT, King TS, Icitovic N, Bleecker ER, Castro M, et al. Comparison of physician-, biomarker-, and symptom-based strategies for adjustment of inhaled corticosteroid therapy in adults with asthma: the BASALT randomized controlled trial. JAMA. 2012;308:987-97.

34. Dweik RA, Sorkness RL, Wenzel S, Hammel J, Curran-Everett D, Comhair S, et al. Use of exhaled nitric oxide measurement to identify a reactive, atrisk phenotype among patients with asthma. Am J Respir Crit Care Med. 2010;181:1033-41.

35. Martin RD. Linking physicians' pay to the quality of care-a major experiment in the United Kingdom. N Engl J Med. 2004;351:1448-54.
36. Fowler SJ, Tavernier G, Niven R. High blood eosinophil counts predict sputum eosinophilia in patients with severe asthma. J Allergy Clin Immunol. 2015;135(822-4):e2.

37. Price D, Ryan D, Burden A, Ziegenweidt JV, Gould S, Freeman D, et al. Using fractional exhaled nitric oxide (FeNO) to diagnose steroid-responsive disease and guide asthma management in routine care. Clin Transl Allergy. 2013;3:37.

38. Malinovschi A, Janson C, Borres M, Alving K. Simultaneously increased fraction of exhaled nitric oxide levels and blood eosinophil counts relate to increased asthma morbidity. J Allergy Clin Immunol. 2016;138(1301-08):e2.

39. National Institute for Health and Care Excellence (NICE). NICE Guidelines: Asthma: diagnosis, monitoring and chronic asthma management. 2017. https://www.nice.org.uk/guidance/ng80. Accessed 12 June 2019.

40. British Thoracic Society. Scottish Intercollegiate Guidelines Network: British guideline on the management of asthma. 2016. https://www. brit-thoracic.org.uk/quality-improvement/guidelines/asthma/. Accessed 12 June 2019

41. Zeiger RS, Yegin A, Simons FE, Haselkorn T, Rasouliyan L, Szefler SJ, et al. Evaluation of the National Heart, Lung, and Blood Institute guidelines impairment domain for classifying asthma control and predicting asthma exacerbations. Ann Allergy Asthma Immunol. 2012;108:81-7.

42. Quezada W, Kwak ES, Reibman J, Rogers L, Mastronarde J, Teague WG, et al. Predictors of asthma exacerbation among patients with poorly controlled asthma despite inhaled corticosteroid treatment. Ann Allergy Asthma Immunol. 2016;116:112-7.

43. International Severe Asthma Registry (ISAR). http://isaregistries.org/. Accessed 18 July 2019.

44. ClinicalTrials.gov. Bethesda (MD): National Library of Medicine (US). Identifier NCT03373045. Observational study of characteristics, treatment and outcomes with severe asthma in the United States (CHRONICLE). 2018. https://clinicaltrials.gov/ct2/show/NCT03373045. Accessed 18 July 2019.

\section{Publisher's Note}

Springer Nature remains neutral with regard to jurisdictional claims in published maps and institutional affiliations.
Ready to submit your research? Choose BMC and benefit from:

- fast, convenient online submission

- thorough peer review by experienced researchers in your field

- rapid publication on acceptance

- support for research data, including large and complex data types

- gold Open Access which fosters wider collaboration and increased citations

- maximum visibility for your research: over 100M website views per year

At BMC, research is always in progress.

Learn more biomedcentral.com/submissions 